\title{
PROTIPUSH PROTEIN POWDER: Effective Supplementation for Strengthen Your Immune System
}

Dr. M.Sampoorna, Madugula Mahender \& Vijaya Bhavani

Besiara Pharmaceuticals, Hyderabad, Ranga Reddy, Telangana, 500058, India.

\section{Corresponding Author}

Dr. M. Sampoorna

DOI: $10.38177 /$ AJBSR.2020.2208

\section{Introduction}

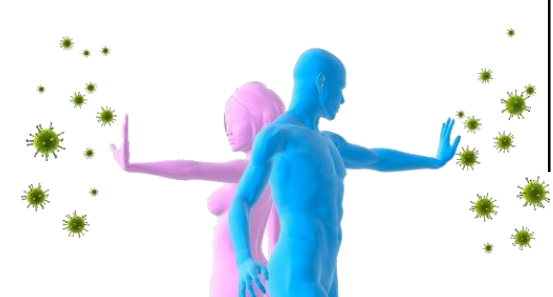

Abstract: Researchers are exploring the effects of diet, exercise, age, psychological stress, and other factors on the immune response, both in animals and in humans. In the meantime, general healthy-living strategies are a good way to start giving your immune system the upper hand. While some people age healthily, the conclusion of many studies is that, compared with younger people, the elderly are more likely to contract infectious diseases and, even more importantly, more likely to die from them. Respiratory infections, influenza, the COVID-19 virus and particularly pneumonia are a leading cause of death in people over 65 worldwide. Scientists have long recognized that people who live in poverty and are malnourished are more vulnerable to infectious diseases. Whether the increased rate of disease is caused by malnutrition's effect on the immune system there is scientific evidence that various micronutrient deficiencies - for example, deficiencies of zinc, selenium, iron, copper, folic acid, and vitamins A, B6, C, E, D3, $K$, Colostrum and Psyllium Husk Etc. alter immune responses.

A vitamin is an organic molecule that is an essential micronutrient which an organism needs in small quantities for the proper functioning of its metabolism. Essential nutrients cannot be synthesized in the organism, either at all or not in sufficient quantities, and therefore must be obtained through the diet.

\section{Vitamin A (Retinol)}

Vitamin A (Vit-A) is a micronutrient that is crucial for maintaining vision, promoting growth and development, and protecting epithelium and mucus integrity in the body. VitA is known as an anti-inflammation vitamin because of its critical role in enhancing immune function. Vitamin A plays a central role in the development and differentiation of white blood cells such as lymphocytes, which play critical roles in immune response.

MOA

Vitamin A can affect the biosynthesis of various proteins, including those involved in regulation of development and cell functioning or determining cell sensitivity to hormones and hormone-like factors. Vitamin A influences the creation of secretory proteins.

\section{Pharmacodynamics}

Vitamin A refers to a group of fat-soluble substances that are structurally related to and possess the biological activity of the parent substance of the group called all-trans retinol or 
retinol. Vitamin A plays vital roles in vision, epithelial differentiation, growth, reproduction, pattern formation during embryogenesis, bone development, hematopoiesis and brain development. It is also important for the maintenance of the proper functioning of the immune system.

\section{Vitamin C (Ascorbic Acid)}

It is a potent antioxidant and a cofactor for a family of biosynthetic and gene regulatory enzymes. Vitamin $C$ contributes to immune defense by supporting various cellular functions of both the innate and adaptive immune system. Thus a vitamin $C$ deficiency results in a

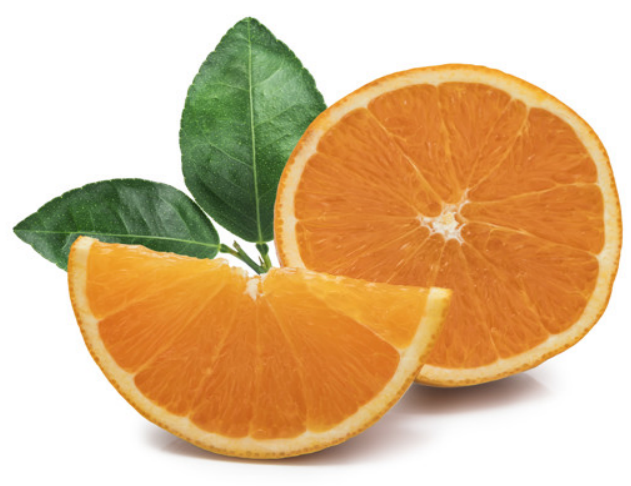
reduced resistance against certain pathogens whilst a higher supply enhances several immune system parameters. Vitamin $\mathrm{C}$ is found in many different fruits and vegetables, including: blackcurrants, citrus fruits - oranges, limes and lemons, berries, kiwifruit, tomatoes, broccoli, sprouts, red, yellow and green capsicum. Vitamin $\mathrm{C}$ has antioxidant properties that aid in tissue building. Vitamin C supports infection-fighting cells and it also promotes healthy skin and wound healing. This vitamin is key in the production of collagen and helps aid the body to fight against disease.

\section{Mechanism}

Vitamin C, also known as ascorbic acid, is necessary for the growth, development and repair of all body tissues. It's involved in many body functions, including formation of collagen, absorption of iron, the immune system, wound healing, and the maintenance of cartilage, bones, and teeth. It is well known that ascorbate acts physiologically as a reluctant and enzyme cofactor.

\section{Vitamin D3 (Cholecalciferol)}

Vitamin $D$ is a fat-soluble vitamin that plays a number of critical roles in your body. That said, some research shows that having healthy levels of vitamin D, as well as taking a vitamin D supplement, can help keep your immune system healthy and may protect against respiratory illnesses in general. Vitamin D can modulate the innate and adaptive immune responses. Deficiency in vitamin D is associated with increased autoimmunity as well as an increased susceptibility to infection. Vitamin D assists in skin repair and helps fight off infection as well as decrease inflammation. 


\section{Vitamin E (Tocopherol)}

Vitamin $E$ is an important nutrient for maintaining the immune system, especially in the aged. Vitamin $E$ is the most important chain-breaking, lipid-soluble antioxidant present in body tissues of all cells and is considered the first line of defense against lipid peroxidation and it is important for normal function of the immune cells.

For the first time, researchers have shown that higher doses of vitamin $E$ can mitigate the stress on immune cells. One sub-class of these cells, the CD8+T cells, eliminates the virus by killing cells it has infected. Other T cells, known as CD4+ T cells, coordinate the immune response to all kinds of pathogens.

\section{Vitamin K (Phytonadione)}

Vitamin $\mathrm{K}$ can act as a cofactor for some plasma proteins, thereby affecting immune and inflammatory responses particularly mediated by $\mathrm{T}$ cells. Vitamin $\mathrm{K}$ plays key roles in different physiological functions such as blood coagulation, bone metabolism, and the regulation of some enzyme systems. It can also act as a cofactor for some plasma proteins, thereby affecting immune and inflammatory responses particularly mediated by $\mathrm{T}$ cells.

\section{Vitamin B}

The ' $\mathrm{B}$ ' complex is meant to be 8 soluble $B$ vitamins compounds of Vitamin 'B' group, which work together as an energy stimulant. B vitamins are necessary for the proper functioning of the methylation cycle, DNA synthesis, and repair and maintenance of phospholipids.

\section{Vitamin B Complex has 8 Vitamins-}

Thiamine (B1), Riboflavin (B2), Niacin (B3), Pantothenic Acid (B5), Pyridoxine (B6), Biotin (B7), Foil (B9) and cobalamin (B12). These are called together as Vitamin 'B' complexes.

\section{Vitamin B1 (Thiamine)}

B1 Vitamin is also called Thiamine. Its main function is to participate in the risk of carbohydrates and take them out of power. Besides, to maintain normal appetite and to keep the nervous system active. B1 also plays an important role in enhancing the performance of the cells. In fact, this vitamin is rich in pulse and hole gran foods.

\section{Vitamin B2 (Riboflavin)}

Vitamin B2, also called riboflavin, is best known for its ability to help the body process proteins, fats and carbohydrates. As well as improving the ability of digestion, it also helps to reduce the incidence of gas-induced and increase the number of blood cells. 


\section{Vitamin B3}

Vitamin B3 stimulate a gene involved in the production of specialized immune cells called neutrophils. Vitamin B4 also known as adenine, vitamin B4 is a water soluble and infamous vitamin. It is a component of DNA, RNA, ATP, and the three coenzymes NAD (nicotinamide adenine dinucleotide), NADH (a reduced form of NAD) and FAD (flavin adenine dinucleotide). Adenine functions synergistically and closely with vitamins B2 and B3 to generate energy. It may enhance antibody formation by the immune system.

\section{Vitamin B5}

Regular use of eggs, nuts, dairy products, broccoli, hole grenades and bins, reduces this vitamin deficiency. B5 plays a special role in the formation of cells.

\section{Vitamin B6 (pyridoxine)}

Vitamin B6 is vital to supporting biochemical reactions in the immune system. It helps in the production of energy. About 100 types of enzyme reactions are justified, keep in mind Vitamin B6. Promoting protein metabolism also accelerates. In fact, these vitamins are abundant in milk, curd, eggs, meat and greens.

\section{Vitamin B7}

Vitamin B7 is the responsibility of keeping hair and nails beautiful as well as enhancing the beauty of B7. Not only that, the metabolism process is justified, but also Vitamin B7. Previous studies have shown that biotin affects the functions of adaptive immune $T$ and NK cells, but its effect(s) on innate immune cells is not known. Biotin status may affect secretion of cytokines by immune cells. These data suggest that biotin supplementation affects gene expression in human immune cells. Effects of biotin on gene expression are likely to modulate the response of immune cells to antigens.

\section{Vitamin B9}

Vitamin B9 (that is, folate or folic acid) is essential for nucleic acid and protein synthesis (40), and inadequate levels of vitamin B9 dramatically alter the immune response. It also helps in the form of complications during pregnancy. That is why experts think that the mothers regularly eat green vegetables, Halen gran food, beans, pulses and banana experts.

\section{Vitamin B12 (cyanocobalamin)}

It helps in the growth and production of red blood cells. Helps in increasing the number of white blood cells and ancillaries. In addition to that there is no disturbance in the function of 
the brain. .B12 plays an important role in white blood cell production, and white blood cells are essential for proper immune system functioning," says Middleburg. Not only can a lack of B12 lower your immunity, some immune system disorders can increase your likelihood of becoming deficient. Vitamins B9 and B12 are dietary nutrients essential for proper maintenance of immune health and cellular function. They contribute to methylation reactions, cellular function and division, DNA synthesis, repair, replication, and production of neurotransmitters.

\section{Calcium}

Calcium acts like a beacon for your immune system. It surrounds foreign matter, marking it for other immune system players, such as phagocytes, to come and destroy. "We found that calcium signals play a vital role in keeping the immune system finely balanced, ramping responses up and down at the appropriate time. Calcium Helps Your Body Fight Infectious Disease. Calcium acts as a second messenger in many cell types, including lymphocytes. Calcium seems to play a central role in the activation of cells of the immune system. When the cells are stimulated, [Ca2+]i generally increases as a result of entry from the external medium, as well as mobilization of calcium from intracellular membrane-bound compartments.

\section{Zinc}

Zinc is crucial for normal development and function of cells mediating innate immunity, neutrophils, and NK cells. Macrophages also are affected by zinc deficiency. Phagocytosis, intracellular killing, and cytokine production all are affected by zinc deficiency. When cold and flu season hits, it's wise to have an arsenal of vitamins at hand to fortify your immune system. Zinc deficiency adversely affects the growth and function of T and B cells. The ability of zinc to function as an anti-oxidant and stabilize membranes suggests that it has a role in the prevention of free radical-induced injury during inflammatory processes. It lowers the inflammation that can often come with a winter cold or the flu.

\section{Iron}

Iron enhances the growth and virulence of pathogens; at the same time, it is essential for the activation and proliferation of immune cells. Therefore, iron deficiency negatively affects the pathogen and the host, albeit more so in the case of host immunity. Since iron is essential for the growth and virulence of most microbial pathogens, it should not be surprising that the mammalian immune system has evolved ways to deprive microorganisms of this vital element. 
Potassium

The amount of potassium disrupts the equilibrium of immune cells within the tumor microenvironment, which then leads to dysfunction in nutrient uptake and membrane charge, which hinders a cell's ability to function properly. You should not take potassium supplements unless your doctor prescribes them. Potassium is necessary for the function of all living cells, and is thus present in all plant and animal tissues.

\section{Phosphorous}

Phosphorous may be considered as part of an integrated approach to support immune functions and maintain a stable microbial ecosystem in the GIT, thereby providing a barrier against potential pathogens. $\mathrm{P}$ is an essential nutrient for both microbes and their host, as it is involved, for example, in bone formation, energy metabolism, cellular signaling and stabilization of cell membranes.

\section{Omega 369 Fatty Acid}

Omega-3 backed to boost immune response, not just battle inflammation: Study. Long chain omega-3 fatty acids may help to boost the immune system by enhancing the functioning of immune cells, according to new research. Omega-3 fats are a crucial part of human cell membranes. They also have a number of other important functions, including: Improving heart health: Omega-3 fatty acids can increase "good" HDL cholesterol. They can also reduce triglycerides, blood pressure and the formation of arterial plaques.

\section{Selenium}

Selenium plays an important role in the health of your immune system. This antioxidant helps lower oxidative stress in your body, which reduces inflammation and enhances immunity. Studies have demonstrated that increased blood levels of selenium are associated with enhanced immune response. Dietary selenium (Se), mainly through its incorporation into selenoproteins, plays an important role in inflammation and immunity. Evidence has emerged regarding roles for individual selenoproteins in regulating inflammation and immunity

\section{Magnesium}

Regarding the relation between $\mathrm{Mg}$ and the immune system, several groups leading in Nutrition and Immunology have shown evidence that magnesium plays a key role in the immune response; that is, as a co-factor for immunoglobulin synthesis, $\mathrm{C}^{\prime} 3$ convertase, immune cell adherence, antibody-dependent cytolysis, IgM. Mg has a strong relation with 
the immune system, in both nonspecific and specific immune response, also known as innate and acquired immune response.

\section{Copper}

Elevated concentrations of copper can be found near the sites of infection. Other studies have shown that copper deficiency in the host can be linked to increased susceptibility to infection (and improved immune response when the host is provided with a copper supplement).Copper is an essential nutrient for the body. Together with iron, it enables the body to form red blood cells. It helps maintain healthy bones, blood vessels, nerves, and immune function, and it contributes to iron absorption. Sufficient copper in the diet may help prevent cardiovascular disease and osteoporosis, too. Oysters and other shellfish, whole grains, beans, nuts, potatoes, and organ meats (kidneys, liver) are good sources of copper. Dark leafy greens, dried fruits such as prunes, cocoa, black pepper, and yeast are also sources of copper in the diet.

\section{Chromium Picolinate}

Chromium is of significant importance in altering the immune response by immunostimulatory or immunosuppressive processes as shown by its effects on $T$ and $B$ lymphocytes, macrophages, cytokine production and the immune response that may induce hypersensitivity reactions. Chromium is a naturally occurring heavy metal found commonly in the environment in trivalent, $\mathrm{Cr}$ (III), and hexavalent, $\mathrm{Cr}$ (VI), forms. $\mathrm{Cr}$ (VI) compounds have been declared as a potent occupational carcinogen among workers in chrome plating, stainless steel, and pigment industries. The reduction of $\mathrm{Cr}$ (VI) to $\mathrm{Cr}$ (III) results in the formation of reactive intermediates that together with oxidative stress oxidative tissue damage and a cascade of cellular events including modulation of apoptosis regulatory gene p53, contribute to the cytotoxicity, genotoxicity and carcinogenicity of $\mathrm{Cr}$ (VI)-containing compounds. On the other hand, chromium is an essential nutrient required to promote the action of insulin in body tissues so that the body can use sugars, proteins and fats.

\section{Sodium}

Salt (sodium chloride) is an important environmental factor that influences various immune cells at biological barriers, including the skin, intestine and kidney. Innate and adaptive immune cells can sense hypertonic sodium in the interstitium, which subsequently affects their differentiation and/or function. Alterations to the intestinal bacterial community induced by excess dietary salt represent another relevant axis whereby salt indirectly modulates immune cell function. 


\section{Manganese}

There is little information regarding the effects of manganese deficiency on immune development and function. There are, however, limited data suggesting that toxic levels of manganese may impair immune function. Limiting the availability of zinc and manganese as a mechanism to defend against infection expands the spectrum of nutritional immunity and further establishes metal sequestration as a key defense against microbial invaders.

\section{Colostrum}

Colostrum may strengthen your immune system and help your body fight disease-causing agents. The immune-boosting effects of colostrum are mostly due to its high concentration of the antibodies IgA and IgG. Antibodies are proteins that fight viruses and bacteria. Cow colostrum is the first milk that is lactated by the cow after delivery. Bovine colostrum is also taken by mouth for boosting the immune system, healing injuries, repairing nervous system damage, improving mood and sense of well-being, slowing and reversing aging, and as an agent for killing bacteria and fungus. Cow colostrum is the first milk that is lactated by the cow after delivery.

\section{Psyllium Husk}

Psyllium is a prebiotic a substance needed for healthy colonies of probiotics to grow in the gut. A healthy colony of good bacteria in the digestive system is essential for healthy immune function. However, research shows that taking psyllium is beneficial to many parts of the human body, including the heart and the pancreas.

\section{Choline}

A new study suggests that a fat-soluble type of choline found mainly in egg yolks, beef and chicken promotes the development of a healthy immune system. "The body's liver produces choline in small amounts.

\section{Conflicts of Interest}

The authors declare that there is no conflict of interest.

\section{References}

1. Jenkins DJ, Spence JD, Giovannucci EL, Kim YI, Josse R, Vieth R, , "Supplemental Vitamins and Minerals for CVD Prevention and Treatment", Journal of the American College of Cardiology. 71 (22): 2570-2584. doi:10.1016/j.jacc.2018.04.020. PMID 29852980.

1. Akhtar S, Ahmed A, Randhawa MA, Atukorala S, Arlappa N, Ismail T, Ali Z (December 2013), "Prevalence of vitamin A deficiency in South Asia: causes, outcomes, and possible 
remedies", Journal of Health, Population, and Nutrition, Vol no: 31, ISSN No: 4, Page no: 413-23.

2. Yetley, Elizabeth A (2007). "Multivitamin and multimineral dietary supplements: definitions, characterization, bioavailability, and drug interactions", The American Journal of Clinical Nutrition, Vol no: 85, ISSN No: 1, Page no: 269S-276S.

3. Khan SU, Khan MU, Riaz H, Valavoor S, Zhao D, Vaughan L, , "Effects of Nutritional Supplements and Dietary Interventions on Cardiovascular Outcomes: An Umbrella Review and Evidence Map", Annals of Internal Medicine, Vol no: 171, ISSN No: 3, Page no: 190198.

4. Holick MF (December 2004). "Sunlight and vitamin D for bone health and prevention of autoimmune diseases, cancers, and cardiovascular disease", The American Journal of Clinical Nutrition, Vol no: 80, (6 Suppl), Page no: 1678S-88S.

5. Holick MF (March 2006). "High prevalence of vitamin D inadequacy and implications for health", Mayo Clinic Proceedings, Vol no: 81, ISSN No: 3, Page no: 353-73.

6. Zetterstrom R (May 2009). "Nobel Prize 1937 to Albert von Szent-Gyorgyi: identification of vitamin C as the anti-scorbutic factor", Acta Paediatrica, Vol no: 98, ISSN no: 5, Page no: 915-9.

7. Rice BH, Quann EE, Miller GD. Meeting and exceeding dairy recommendations, effects of dairy consumption on nutrient intakes and risk of chronic disease, Nutr Rev, 2013, Vol no:71, Page no: 209-223.

8. Pasiakos SM. Exercise and amino acid anabolic cell signaling and the regulation of skeletal muscle mass, Nutrients, 2012, Vol no: 4, Page no: 740-758.

9. van den Bergh JJ, Bidar SS, Bours S, van Geel TA, Geusens PP, Need of calcium and vitamin $D$ in patients after recent fracture, Food Nutr Sci, 2012, Vol no: 3, Page no: 539547.

10. Rice BH. Dairy and cardiovascular disease: a review of recent observational research, Curr Nutr Rep, 2014, Vol no: 3, Page no: 130-138.

11. Huth PJ, Park KM. Influence of dairy product and milk fat consumption on cardiovascular disease risk: a review of the evidence, Adv Nutr, 2012, Vol no: 3, Page no: 266-285.

12. Weaver CM. Calcium supplementation: is protecting against osteoporosis counter to protecting against cardiovascular disease, Curr Osteoporos Rep, 2014, Vol no: 12, Page no: 211-218. 
13. Rappuoli R, Pizza M, Del Giudice G, De Gregorio E, Vaccines, new opportunities for a new society, Proc Natl Acad Sci USA, (2014), Vol no: 111, Page no: 12288-93.

14.Davis DM. Presenting the marvels of immunity, Nat Rev Immunol, (2014), Vol no: 14, Page no: 351-3.

15. https://www.health.harvard.edu/staying-healthy/how-to-boost-your-immune-system.

16. Pauling L. Ascorbic acid and the common cold, Am J Clin Nutr, (1971), Vol no: 24, Page no: $1294-9$. 